\title{
Electrical Equivalent Circuit of Multi-Tips Pulsed Corona Discharge Reactor
}

\author{
Karim Saber ${ }^{\mathrm{a}}$, Alyen Abahazem ${ }^{\mathrm{a}, \mathrm{c}}$, Nofel Merbahi ${ }^{\mathrm{b}}$, Mohamed Yousfi ${ }^{\mathrm{b}}$ \\ ${ }^{a}$ Laboratory Materials and Renewable Energies, Physics Department, Cité Dakhla BP 8106, Ibn Zohr University, Agadir, Morocco \\ ${ }^{b}$ LAPLACE UMR 5213-CNRS, 118 Route de Narbonne, Bât. 3R2, 31062 Toulouse Cedex 9, Paul Sabatier University, France \\ CLASDIS, CRMEF-SM, Avenue Mly Abdellah, BP 106, Inezgane, Morocco
}

\begin{abstract}
In this work, an electrical model equivalent to the corona discharge reactor has been proposed in a multitips plan configuration, in dry air at atmospheric pressure. The electrical parameters evolution of the circuit are obtained by using the identification method which is based on the least squares recursive (RLS) algorithm, the estimated parameters allow us to describe the corona discharge behavior inside the reactor. The RLS method used during the determination of capacitance and resistance is validated by the comparison between the measured and the calculated currents, the significant forms of capacitance and resistance confirm the validity of the proposed electrical model. The estimated parameters of the electrical circuit allowed us to determine the discharge power, the power delivered to the reactor and thus the energy efficiency during the discharge, this efficiency increases during the propagation of streamers towards the plane, it reaches a maximum value which is equal to $50 \%$ in the case of the fourtips-plane configuration. The energy stored in the reactor is also calculated using the electrical circuit, it increases to a maximum value of $2.6 \mathrm{pJ}$, which is a very low value compared to the energy delivered to the reactor. This work allows us to control the discharge and lost energy during the corona discharge in the case of multi-tips-plane configuration.
\end{abstract}

Keywords: Corona discharge; Voltage measurement; Current measurement; electrical model; discharge power; delivered power; Plasma energy efficiency. 